\title{
Randomised comparison of electrode positions for cardioversion of atrial fibrillation
}

T P Mathew, A Moore, M McIntyre, M T Harbinson, N P S Campbell, A A J Adgey, G W N Dalzell mining the success rate and energy requirement have not been conclusively established.

Cardioversion occurs when a critical mass of atrial muscle is depolarised by transthoracic current. The current flow is dependent on the energy level selected and the transthoracic impedance (TTI). Several factors are known to influence the TTI: factors related to the defibrillator (shock strength), electrode system (electrode pad size, pad position, interface between the electrode and the skin), anthropometric factors (body mass index, percentage of body fat, chest anteroposterior diameter), or those related to the technique (time intervals between shocks, phase of ventilation, paddle electrode pressure, previous sternotomy)..$^{2-9}$

Optimal electrode position is important because a critical mass of atrial muscle must be depolarised for cardioversion to occur. Some investigators have suggested the anteroposterior (AP) pad position may be more effective than the conventional anteroanterior (AA) position. ${ }^{10-12}$ We and others have previously reported lower TTI and hence higher generated current in the AP position. ${ }^{413}$ Lown et al found a higher success rate with low energy shocks in the AP pad position. ${ }^{11}$ A computerised tomographic analysis of the thorax suggested that the current vector traverses a maximum mass of atrial muscle with electrodes in the AP electrode position. ${ }^{10}$ There has been only one study that directly compared AP with AA pad positions, although this was not prospectively randomised and used hand held metal paddles instead of the self adhesive electrode pads now in common use. ${ }^{13}$

We therefore compared the success rate and energy requirements for cardioversion of atrial fibrillation using AP and AA electrode pad positions with self adhesive ECG/defibrillator pads.

Regional Medical Cardiology Centre, Royal Victoria Hospital, Belfast BT12 6BA, Northern Ireland, UK T P Mathew A Moore

M McIntyre

M T Harbinson

N P S Campbell

A A J Adgey

G W N Dalzell

Correspondence to: Dr Mathew.

email: rmcc@pipex.dial.com

Accepted for publication 15 January 1999 for the first shock (AA 77.5 (18.4) ohms; AP $73.7(18.7)$ ohms; $p=0.34)$ was not significantly different between the two groups. TTI correlated significantly with body mass index, percentage body fat, and chest AP diameter. There was a progressive decrease in TTI with serial shocks. While aetiology and TTI were the two independent significant predictive factors for energy requirement, duration of atrial fibrillation was the only independent predictor of cardioversion success in a multivariate analysis.

Conclusions-Electrode pad position is not a determinant of cardioversion success rate or energy requirement.

(Heart 1999;81:576-579)

Keywords: atrial fibrillation; cardioversion; electrode pad position

Electrical cardioversion has been a successful technique for the treatment of atrial fibrillation ever since its introduction in the $1960 \mathrm{~s}^{1}{ }^{1}$ However, the relative importance of factors deter-

\section{Methods}

Ninety consecutive patients undergoing elective cardioversion for atrial fibrillation were prospectively randomised to AA or AP pad positions. Patients with a permanent pacemaker in situ were excluded. The duration of atrial fibrillation was determined as the time from the first ECG documentation. Echocardiography was performed within the previous six months in 82 patients and the left atrial diameter recorded. Height and weight of all patients were recorded on the day of the procedure and body mass index calculated. Percentage body fat was measured using a bioimpedance machine (Bodystat Ltd, Douglas, Isle of Man, UK). Patients were fasted 
overnight, digoxin was omitted for 24 hours, and sedation with intravenous midazolam was given. Self adhesive ECG/defibrillator pads, each with an area of $106 \mathrm{~cm}^{2}$, were attached to the chest wall. For the AA position the electrode pads were placed in the right infraclavicular region and at the cardiac apex; for the AP position pads were placed in the right infraclavicular and left infrascapular region.

TTI was measured using a low amplitude current $(18 \mathrm{kHz})$ passed across the patient's chest between the self adhesive pads. The resultant direct current voltage is proportional to the TTI. We and others have previously validated the accuracy of this method using a 30 $\mathrm{kHz}$ current. ${ }^{14}{ }^{15}$ The peak current generated for each shock was recorded.

The energy protocol was an initial shock of $100 \mathrm{~J}$ which, if unsuccessful, was followed by $200 \mathrm{~J}, 300 \mathrm{~J}$, and $360 \mathrm{~J}$ if required. In the last 10 patients, if the initial $360 \mathrm{~J}$ shock was unsuccessful in one position cardioversion was attempted by the other route using a further $360 \mathrm{~J}$ shock; in these 10 patients TTI was recorded in both positions.

\section{STATISTICAL ANALYSIS}

All results for continuous variables are expressed as means (SD). The independent sample $t$ test was used to compare continuous variables between the two subgroups. The $p$ values for comparison of categorical variables were generated by the Pearson $\chi^{2}$ for proportions with appropriate degrees of freedom, and $\mathrm{p}$ values $<0.05$ were taken as significant. Multivariate analysis was done using forward stepwise (likelihood ratio) multiple regression analysis. All calculations were carried out with SPSS 7.1 software package.

\section{Results}

Forty five patients were randomised to each position. There were 60 male and 30 female patients with a mean (SD) age of 65.5 (10) years. The mean duration of atrial fibrillation was 14.1 (31.7) months and the mean left atrial dimension was $48.7(8.6) \mathrm{mm}$. The aetiology of the atrial fibrillation was ischaemic heart disease in $28(31 \%)$, hypertensive heart disease in $19(21 \%)$, rheumatic heart disease in 15 $(16 \%)$, post-cardiac surgery in seven $(7 \%)$, others in $21(23 \%)$.

Cardioversion was successful in $81 \%$ of the patients (73/90). A mean of 2.4 (1.1) shocks was delivered to each patient, and the average energy of the successful shock (or maximum if unsuccessful) was 227.6 (102.8) J. The mean cumulative energy delivered per patient was 452 (331.1) J (range $100 \mathrm{~J}$ to $1320 \mathrm{~J}$ ). Low energy shocks $(\leqslant 200 \mathrm{~J})$ were successful in $58 \%$ of the patients $(52 / 90)$.

The mean TTI for the first shock was 75.6 (18.5) ohms (range 43 to $133 \mathrm{ohms}$ ). This significantly correlated with the body mass index, percentage body fat, and chest AP diameter. There was a progressive decrease in TTI with serial shocks, and the mean TTI for the final shock was $68.8(15.4)$ ohms $(\mathrm{p}=0.001)$.
Table 1 Baseline characteristics according to pad position

\begin{tabular}{llll}
\hline & $A A$ & $A P$ & $p$ Value \\
\hline BMI $\left(\mathrm{kg} / \mathrm{m}^{2}\right)$ & $27.5(4.9)$ & $26.5(5.3)$ & NS \\
AP diameter (cm) & $25.9(3.3)$ & $25.2(3.9)$ & NS \\
AF duration (months) & $14.7(40.2)$ & $13.3(20.4)$ & NS \\
Percentage body fat & $29.5(8.9)$ & $31.2(9.4)$ & NS \\
LA dimension (mm) & $48.9(7.2)$ & $48.4(9.8)$ & NS \\
\hline
\end{tabular}

$\mathrm{AA}$, anteroanterior; $\mathrm{AF}$, atrial fibrillation; $\mathrm{AP}$, anteroposterior; BMI, body mass index; LA, left atrium. Values are mean (SD).

Table 2 Transthoracic impedance and energy requirements according to electrode pad position

\begin{tabular}{llll}
\hline & $A A$ & $A P$ & $p$ Value \\
\hline Mean energy (J) & $223(96.1)$ & $232(110)$ & NS \\
TTI, first shock (ohm) & $77.5(18.4)$ & $73.7(18.7)$ & NS \\
TTI, final shock (ohm) & $72.2(17.1)$ & $65.3(13.0)$ & 0.03 \\
Peak current (amp) & $35.7(9.3)$ & $37.4(11.7)$ & NS \\
Number of shocks & $2.3(1.1)$ & $2.4(1.2)$ & NS \\
Cumulative energy (J) & $432(314)$ & $472(350)$ & NS \\
\hline
\end{tabular}

$\mathrm{AA}$, anteroanterior; AP, anteroposterior; TTI, transthoracic impedance. Values are mean (SD).

Comparison of baseline characteristics revealed no statistically significant difference between patients randomised to either position (table 1). Cardioversion success rate was not significantly different between the two pad positions (AA 84\%, 38/45 patients; AP 78\%, $35 / 45$ patients; $p=0.42$ ). The energy requirements were not significantly different, as $62 \%$ of patients (28/45) with pads in the AA position were successfully cardioverted with shocks of $\leqslant 200$ J compared with $53 \%(24 / 45)$ of those with pads in the AP position $(p=0.39)$. The mean energy of the successful or final shock was not different between the two groups. Mean peak current, first shock TTI, and mean cumulative energy for all patients were also similar (table 2).

For patients who were successfully cardioverted, TTI was lower in the AP position although this was not statistically significant (AA 77.4 (17.7) ohms; AP 72.5 (17.1) ohms; $\mathrm{p}=0.2$ ). The mean energy (AA 197.9 (82.4) J; AP $195.4(97.2) \mathrm{J} ; \mathrm{p}=0.9)$ and peak current (AA 33.6 (7.9) amp; AP 33.7 (10.5) amp; $\mathrm{p}=0.9)$ were similar. When cardioversion success rate was analysed at each energy level, there was no significant difference in success rate between the two positions (fig 1 ).

To predict cardioversion success and energy requirement, the following factors were entered into a forward stepwise (likelihood ratio) multiple regression analysis: age, aetiology, drug

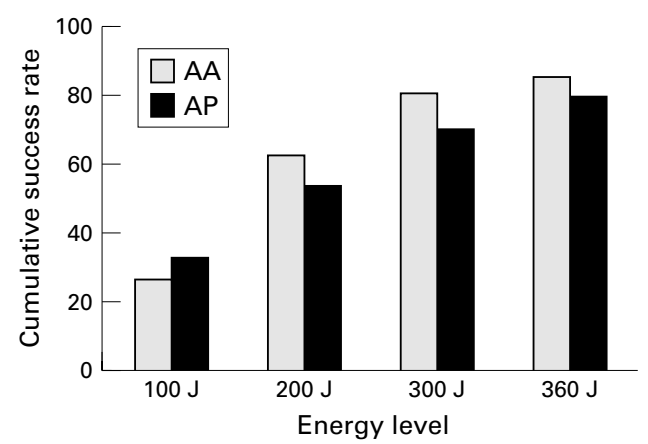

Figure 1 Cumulative cardioversion success rate at each energy level according to pad position. AA, anteroanterior; $A P$, anteroposterior. 
treatment, duration of atrial fibrillation, left atrial dimension, pad position, and TTI. The only significant determinant of successful cardioversion was duration of atrial fibrillation-shorter durations were more likely to be successfully cardioverted. For energy requirement, TTI and aetiology were significant independent predictors-patients with lower TTI and atrial fibrillation secondary to rheumatic heart disease and following open heart surgery were more often cardioverted with low energy shocks.

In the last 10 patients TTI was measured in both positions. There was no significant difference in mean TTI between the two pad positions (AA 71.0 (9.7); AP 70.1 (9.0); $\mathrm{p}=0.83$ ). In two of these 10 patients who were randomised to the AP position the initial $360 \mathrm{~J}$ shock was unsuccessful and they therefore crossed over to AA position for a further $360 \mathrm{~J}$ shock, which was also unsuccessful.

\section{Discussion}

Our results suggests electrode position is not a determinant of cardioversion success or energy requirement. These results are similar to those of Kerber et al and Resnekov and McDonald, although neither of those studies was prospectively randomised. ${ }^{13} 16$ The overall cardioversion success rate of $81 \%$ (73/90) compares favourably with other studies. ${ }^{47-19}$ Duration of atrial fibrillation was the only significant independent predictor of cardioversion success. This association between duration of atrial fibrillation and success has been reported previously by other investigators. ${ }^{4} 16172021$

A first shock of $100 \mathrm{~J}$ was successful in $30 \%$ of patients $(27 / 90)$ and low energy shocks $(\leqslant 200 \mathrm{~J})$ were successful in $58 \%(52 / 90)$. TTI and aetiology were both significant independent predictors of energy requirement whereas cardioversion route was not. Patients with high TTI more often required high energy shocks. This is similar to the observation made by Kerber et $a l^{22}$ It is interesting to note that all seven patients with atrial fibrillation following cardiac surgery, 13 of $15(87 \%)$ with atrial fibrillation secondary to rheumatic heart disease, and 12 of $21(58 \%)$ with atrial fibrillation secondary to miscellaneous conditions (cardiac failure, hyperthyroidism, alcohol) converted with low energy shocks, compared with 13 of 28 (46\%) with ischaemic heart disease and seven of 19 (37\%) with hypertension.

The aetiology of atrial fibrillation has previously been suggested as an important determinant of cardioversion success rate and energy requirement by other investigators. Idiopathic atrial fibrillation has been reported to have both poor and good short term results following cardioversion. ${ }^{402324}$ In the present study the group of 21 patients with an aetiology of "other" included 10 patients with idiopathic atrial fibrillation; only six of these were successfully cardioverted, but five of the six required $\leqslant 200 \mathrm{~J}$. Patients with rheumatic heart disease and severe mitral regurgitation or with a large fibrotic atrium following valvar surgery have been reported to have low cardioversion success rates. ${ }^{25}{ }^{26}$ However, we have previously reported success rates in patients with rheumatic heart disease similar to those in patients with other aetiologies, ${ }^{4}$ and similar results were observed in the present study. Fourteen of the 15 patients with rheumatic heart disease $(93 \%)$ were successfully cardioverted with only $13 \%$ (two of 15 ) requiring high energy shocks. All seven patients with atrial fibrillation following open heart surgery were successfully cardioverted with $100 \mathrm{~J}$ shocks. This is in accord with our previous results. ${ }^{4}$ The inconsistent results observed by various investigators suggest that aetiology is not a primary factor in determining the success rate or energy requirement, but may be confounded by the duration of atrial fibrillation. The high success rate and low energy requirement for postoperative atrial fibrillation may reflect the shorter duration of the atrial fibrillation and the lower TTI reported following sternotomy. ${ }^{9}$

The "low energy low efficacy" observation has prompted investigators to recommend an initial shock of $200 \mathrm{~J}$ for cardioversion of atrial fibrillation. ${ }^{27-29}$ The observations made by Ricard et al and our own results suggest that atrial fibrillation of short duration and following open heart surgery have excellent results with $100 \mathrm{~J}$ shocks. ${ }^{20}$ However, defibrillator induced myocardial damage is well recognised and is dose related. ${ }^{30}$ Thus we believe it is inappropriate to recommend initial shock of $200 \mathrm{~J}$ for all patients, as even this energy level can be associated with adverse effects. ${ }^{31}$

As we reported before, TTI was lower in the AP pad position (though not significantly so), and this was associated with a correspondingly higher current in the AP position, although insufficient to produce a higher success rate. ${ }^{5}$ TTI progressively decreased with serial shocks in our study, as observed by others, probably because of ionisation of the thoracic tissue. ${ }^{7}$

Although there are no data to suggest higher cardioversion success rates in any position, some investigators recommend an alternative route if cardioversion fails in one pad position. ${ }^{32}$ The only variable that may change by relocating the pads is the TTI. It is of interest that we did not observe any significant difference in the TTI in the same 10 patients between the AA and AP positions, and the two patients who failed in one position did not succeed in the other. However, the current vector may travel differently in an individual patient in a particular position and this should be evaluated in a larger subset of patients.

1 Lown B. New method for terminating cardiac arrhythmias. FAMA 1962;3:548-55.

2 Ewy GA. Cardioversion. In: Ewy GA, Bressler R, eds. Cardiovascular drugs and management of heart disease. New York: Raven Press, 1982:427-40.

3 Kerber RE, Grayzel J, Hoyt R, et al. Transthoracic resistance in human defibrillation. Influence of body weight, chest size, serial shocks, paddle size and paddle contact pressure. Circulation 1981;63:676-82.

4 Dalzell GWN, Anderson J, Adgey AAJ. Factors determining success and energy requirements for cardioversion of atrial fibrillation: revised version. Q F Med 1991;78:85-95.

5 Aylward PE, Kielso R, Hite P, et al. Defibrillator electrodechest wall coupling agents: influence on transthoracic 682-6.

6 Dalzell GWN, Cunningham SR, Magee H, et al. Human transthoracic impedance-role of skinfold thickness and body fat [abstract]. PACE 1988;11:856. 
7 Dahl CF, Ewy GA, Ewy MD, et al. Transthoracic impedance to direct current discharge: effect of repeated impedance to direct current discharge: effect

8 Ewy GA, Hellman DH, McClung S, et al. Influence of ventilation phase on transthoracic impedance and defibrillation effectiveness. Crit Care Med 1980;8:164-6.

9 Kerber RE, Vance S, Schomer SJ, et al. Transthoracic defibrillation: effect of sternotomy on chest impedance. $\mathscr{F}$ Am Coll Cardiol 1992;20:94-7.

10 Ewy GA. Optimal technique for cardioversion of atrial fibrillation. Circulation 1992;86:1645-7.

11 Lown B, Kleiger R, Wolff G. The technique of cardioversion. Am Heart f 1964; 67:282-3.

12 Morris JJ, Kong Y, North WC, et al. Experience with cardioversion of atrial fibrillation and flutter. Am f Cardiol 1964;14:94-100.

13 Kerber RE, Jensen SR, Grayzel J, et al. Elective cardioversion: influence of paddle-electrode location and size on success rates and energy requirements. $N$ Engl $尹$ Med 1981;305:658-62.

14 Dalzell GWN, Magee H, Anderson J, et al. Predicted and actual impedance in cardioversion [abstract]. Circulation 1986:74(suppl II):II-388.

15 Kerber RE, Kouba C, Martins J, et al. Advance prediction of transthoracic impedance in human defibrillation and cardioversion: importance of impedance in determining the success of low-energy shocks. Circulation 1984;70:303-8.

6 Resnekov L, McDonald L. Appraisal of electroconversion in treatment of cardiac dysrhythmias. Br Heart $\mathcal{F}$ 1968;30 786-811.

17 Pantridge JF, Halmos PB. Conversion of atrial fibrillation by direct current counter shock. Br Heart F 1965;27:128-30.

18 Rabbino MD, Likoff W, Dreifus LS. Complications and limitations of direct current countershock. $\mathcal{F A M A} 1964$; 190;417-20.

19 Johnston SD, Trouton TG, Wilson C. A review of direct current cardioversions for atrial arrhythmia. Ulster Med $\mathcal{F}$ 1998;67:19-24.

20 Ricard P, Levy S, Trigano J, et al. Prospective assessment of the minimum energy needed for external electrical cardiothe minimum energy needed for external electrical cardio-
21 Van Gelder IC, Crijns HJ, Van Gilst WH, et al. Prediction of uneventful cardioversion and maintenance of sinus rhythm from direct current electrical cardioversion of chronic atrial fibrillation and flutter. $A m \mathcal{F}$ Cardiol 1991;68:41-6.

22 Kerber RE, Martins JB, Kienzle MG, et al. Energy, current and success in defibrillation and cardioversion: clinical studies using an automated impedance-based method of energy adjustment. Circulation 1988;77:1038-46.

23 McCarthy C, Varghese PJ, Barritt DW. Prognosis of atrial arrhythmias treated by electrical counter shock therapy. A three year follow up. Br Heart f 1969;31:496-500.

24 Resnekov L, McDonald L. Electroversion of lone atrial fibrillation and flutter including hemodynamic studies at rest and on exercise. Br Heart f 1971;33:339-50.

25 Levy S. Electrophysiology, pacing and arrhythmia. Clin Cardiol 1992;15:445-9.

26 Lown B. Electrical reversion of cardiac arrhythmias. $\mathrm{Br}$ Heart $\mathcal{f}$ 1967;29:469-88.

27 Walters MI, Wright M, Lim R, et al. Cardioversion of atrial fibrillation: is it time to review current guidelines? [abstract] Eur Heart $\mathcal{F}$ 1998;19:491.

28 Walters MI, Nobbs A, Chakraborthy R, et al. Cardioversion of atrial fibrillation: does it matter what initial energy we use? [abstract] Br Heart F 1998;79:P7

29 Walters MI, Norell MS. Energy considerations for the electrical cardioversion of atrial fibrillation. Br f Cardiol 1997; 4:177-8.

30 Van Vleet JF, Tacker WA. Cardiac damage from transchest and ICD defibrillator shocks. In: Tacker WA, ed. Defibrillation of the heart. St Louis: CV Mosby, 1994:259-98.

31 Stoddard MF, Labovitz AJ, Stevens LL, et al. Effects of electrophysiologic studies resulting in electrical countershock or burst pacing on left ventricular systolic and diastolic function. Am Heart f 1988;116:364-70.

32 Kerber RE. Transthoracic cardioversion of atrial fibrillation and flutter: standard techniques and new advances. $A m \mathcal{F}$ Cardiol 1996;78:22-6. 